\title{
Member Characteristics
}

This appendix provides evidence that characteristics of members that were selected to receive a service request, are uncorrelated with the type of profile that was used to send the request. To test this, we ran a probit regression of the profile type (a dummy variable equal to $1(0)$ for a serving (neutral) profile) on the available characteristics of the members that are selected to receive a request. Table S6-1 gives the marginal effects derived from this regression.

Table S6-1: Probit Regression

\begin{tabular}{|c|c|c|}
\hline & \multicolumn{2}{|c|}{ Serving Profile } \\
\hline male & 0.028 & 0.34 \\
\hline age/100 & 0.006 & 0.54 \\
\hline \#providers' references & 0.001 & 0.09 \\
\hline \#travelers' references & 0.011 & 1.21 \\
\hline \#friends & -0.008 & $1.75^{*}$ \\
\hline able to provide & -0.055 & 0.70 \\
\hline days & 0.002 & 0.07 \\
\hline \#observations & \multicolumn{2}{|c|}{189} \\
\hline
\end{tabular}

Notes. The first number in a cell denotes the marginal effect derived from the probit regression of the variable depicted in the row; the second number gives the corresponding z-value. "Serving profile" $=1(0)$ if the request was sent from a serving (neutral) profile. The gender and age refer to the member who received the request. "\#providers' references" denotes the number of references left by other members that have previously provided the service to the member to whom we sent a service request. "\#travelers' references" denotes the number of references left by other members that have previously received the service from the member to whom we sent a service request. "\#friends" denotes the number of friends on the profile of the member to whom we sent a service request. "able to provide" $=1(0)$ if the profile indicates the availability to offer the service as "yes" ("maybe") (recall that no request can be sent to a member indicating "no"). "Days" indicates the number of days between submission of the request and the day for which the service was requested.

*denotes statistical significance at the $10 \%$ level.

The results show very small effects of members' characteristics on whether the request is received from a serving profile or a neutral profile. One out of the seven independent variables is statistically significant at the $10 \%$-level, but even here the marginal effect is very small ( $0.8 \%$-points). 